\title{
Combination of celecoxib and PD184161 exerts synergistic inhibitory effects on gallbladder cancer cell proliferation
}

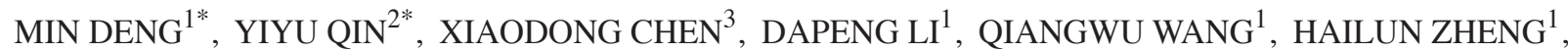 \\ LIN GU $^{1}$, CHAOJING DENG ${ }^{1}$, YONGJU XUE ${ }^{1}$, DANYU ZHU ${ }^{1}$, QIZHI WANG ${ }^{1}$ and JIANCHAO WANG ${ }^{1}$ \\ ${ }^{1}$ Department of Gastroenterology, The First Affiliated Hospital of Bengbu Medical College, \\ Bengbu, Anhui 233004; ${ }^{2}$ Clinical Medical College, Research Centre of Biomedical Technology, \\ Yancheng Institute of Health Sciences, Yancheng, Jiangsu 224005; ${ }^{3}$ Department of Orthopedics, \\ The First Affiliated Hospital of Bengbu Medical College, Bengbu, Anhui 233004, P.R. China
}

Received July 27, 2015; Accepted October 24, 2016

DOI: $10.3892 / \mathrm{ol} .2017 .5914$

\begin{abstract}
Cyclooxygenase-2 (COX-2) and extracellular signal-regulated kinase $1 / 2($ ERK1/2) may serve as potential targets in various types of cancer; however, the roles of these proteins in gallbladder carcinoma (GBC) have not been reported previously. In the present study, the expression levels of COX-2 and phospho (p)-ERK1/2 in GBC were examined and the biological activities of celecoxib and PD184161 (specific inhibitors of COX-2 and p-ERK1/2, respectively) on the proliferation, cell cycle and apoptosis of the GBC-SD and NOZ human GBC cell lines were evaluated by a series of in vitro and in vivo studies. COX-2 and p-ERK1/2 protein expression levels were found to be significantly elevated in GBC tissues as well as in GBC-SD and NOZ cells. Treatments with celecoxib and PD184161 significantly inhibited GBC-SD and NOZ cell growth in a concentration-dependent manner, and their combination produced a synergistic inhibitory effect. In addition, celecoxib and PD184161 significantly inhibited tumor growth in xenograft nude mice. Celecoxib treatment led to G1 arrest via the upregulation of p21 and p27 expression in GBC-SD and NOZ cells, whereas PD184161 did not affect cell cycle distribution. The combination of celecoxib and PD184161 was able to promote cell apoptosis by triggering a collapse of mitochondrial membrane potential and activating caspase-3-mediated apoptosis. In conclusion, COX-2 and p-ERK1/2 protein may serve as potential targets for GBC chemotherapy, and the combination of celecoxib and
\end{abstract}

Correspondence to: Dr Qizhi Wang or Dr Jianchao Wang, Department of Gastroenterology, The First Affiliated Hospital of Bengbu Medical College, 287 Changhuai Road, Bengbu, Anhui 233004, P.R. China

E-mail: 78368922@qq.com

E-mail: 47814286@qq.com

*Contributed equally

Key words: gallbladder carcinoma, celecoxib, PD184161, apoptosis
PD184161 could significantly inhibit GBC cell growth, induce cell G1 arrest and trigger cell apoptosis of GBC cells.

\section{Introduction}

Gallbladder carcinoma (GBC) is the most common malignancy of the biliary system and the fifth most common gastrointestinal cancer worldwide, with an annual incidence of $>10,000$ and mortality rate of $\sim 3,300$ individuals. Its morbidity varies with racial, ethnic and regional factors (1). In recent years, numerous studies have reported an increase in the incidence of GBC in certain areas, including north India, Pakistan and Korea $(2,3)$. As the majority of GBC patients are diagnosed when the cancer has reached an advanced stage, they have an extremely poor prognosis, with a median 5-year survival rate of $2-5 \%$ (4). Chemotherapy is considered to be the most valuable treatment to prolong the survival time and improve the quality of life of these patients. However, GBC shows resistance to the majority of currently used chemotherapeutic agents, and the real clinical effect of such agents is unsatisfactory (5). With rapid developments in cancer molecular and cell biology, targeted cancer therapies, which are drugs that interfere with specific molecules involved in cancer cell growth and survival, bring new hope for cancer patients. The growth and development of most tumors involves multiple genetic abnormalities, which diminishes the antitumor activities of the majority of single-targeted drugs in clinical applications. To date, GBC clinical trials have demonstrated that most single-targeted drugs for GBC have poor specificity and sensitivity (6). Network models suggest that partial inhibition of a surprisingly small number of targets can be more efficient than the complete inhibition of a single target. Therefore, researchers have begun to explore novel multi-targeted drugs and strategies for the combination of targeted drugs or traditional chemotherapy drugs (7).

The overexpression of cyclooxygenase-2 (COX-2), which is a rate-limiting enzyme in prostaglandin production, is involved in the tumorigenesis of various types of tumors, including GBC $(8,9)$. Extracellular signal-regulated kinase $1 / 2($ ERK1/2) is a member of the mitogen-activated protein 
kinase (MAPK) cascade, which is involved in regulating biological processes such as cell growth and division, as well as apoptosis. Several studies have reported that phospho (p-)ERK1/2 expression is abnormally elevated in GBC tissues $(10,11)$, and that aberrant ERK1/2 activation is closely associated with tumorigenesis and progression in various tumor types $(12,13)$. It has also been demonstrated that COX-2 and ERK1/2 may serve as potential therapeutic targets for certain tumors, and their specific inhibitors have shown encouraging outcomes for cancer patients (14). For instance, patients with EGFR wild-type non-small cell lung cancer showed an increased progression-free survival following treatment with a combination of erlotinib and celecoxib (15). However, the biological functions of COX-2 inhibitors and ERK1/2 inhibitors against the growth of GBC cells have not yet been investigated.

In the present study, the biological effects of the COX-2 inhibitor celecoxib and the ERK1/2 inhibitor PD184161 on the growth, cell cycle and apoptosis of the GBC cell lines GBC-SD and $\mathrm{NOZ}$ were examined in vitro and in vivo. Additionally, the molecular mechanisms underlying these activities were preliminarily investigated. This data may provide a theoretical foundation and initial evidence for exploring novel therapeutic regimens for $\mathrm{GBC}$ patients.

\section{Materials and methods}

Sample collection. The present study was approved by the ethics committee of The First Affiliated Hospital of Bengbu Medical College (Bengbu, China), and all patients provided informed consent. Cancer tissue specimens were obtained from 24 patients with GBC who underwent radical cholecystectomy without prior radiotherapy or chemotherapy between June 2006 and May 2009 at the First Affiliated Hospital of Bengbu Medical College. Normal tissues were reserved in $10 \mathrm{GBC}$ cases (all at stage I and II) and were used as negative controls. The specimens were stored in liquid nitrogen immediately following surgery. COX-2 and p-ERK1/2 expression levels in tissues were examined using western blotting.

Cell culture. The human GBC cell line GBC-SD was obtained from the Cell Bank of the Chinese Academy of Sciences (Shanghai, China). The human GBC cell line NOZ was obtained from the Japanese Collection of Research Bioresources Cell Bank (Osaka, Japan). The non-immortalized human biliary epithelial cell line HIBEpiC was obtained from the ScienCell Research Laboratories (Carlsbad, CA, USA). All cell lines were cultured in Gibco Dulbecco's modified Eagle's medium (Thermo Fisher Scientific, Inc., Waltham, MA, USA) supplemented with $10 \%$ fetal bovine serum (GE Healthcare Life Sciences, Logan, UT, USA) and 1\% penicillin-streptomycin (Sigma-Aldrich; EMD Millipore, Billerica, MA, USA). Cells were cultured in a humidified atmosphere of $5 \% \mathrm{CO}_{2}$ at $37^{\circ} \mathrm{C}$. Trypsin $(0.25 \%)$ was used to detach the cells from the culture flask.

Western blotting. Total protein was extracted from cells using a Novagen Protein Extraction Kit (EMD Millipore). The protein extracts were denatured by boiling at $95^{\circ} \mathrm{C}$ for 5 min and equal amounts of proteins were separated on $10 \%$
SDS-PAGE gels and transferred to polyvinylidene difluoride membranes (EMD Millipore). Blots were blocked with 5\% non-fat dry milk and then incubated with the primary antibodies at $4{ }^{\circ} \mathrm{C}$ overnight. The primary antibodies included anti-COX-2 (1:100; sc-514489), anti-p-ERK1/2 (1:200; sc-23759-R), anti-p21 (1:200; sc-6246), anti-p27 (1:200; sc-71813), anti- $\beta$-actin (1:200; sc-47778), anti-caspase-3 (1:200; sc-7148), anti-cytochrome $c(1: 200$; sc-65396) and anti-COX IV (1:100; sc-376731) antibodies (all Santa Cruz Biotechnology, Inc., Dallas, TX, USA). COX IV was used as the loading control for the mitochondrial fraction and $\beta$-actin was used as the loading control for the cytosolic fraction. Subsequently, the membranes were incubated with horseradish peroxidase-conjugated anti-mouse (1:2,000; sc-2005; Santa Cruz Biotechnology, Inc.) and anti-rabbit (1:5,000; sc-2004; Santa Cruz Biotechnology, Inc.) secondary antibodies at room temperature for $1 \mathrm{~h}$. Subsequently, the blots were visualized using Pierce ECL Western Blotting Substrate (Thermo Fisher Scientific, Inc.). $\beta$-actin was used as a loading control.

Cell viability assay. The viability of GBC-SD and NOZ cells was determined by the water soluble tetrazolium (WST)-1 method using a WST-1 Cell Proliferation and Cytotoxicity Assay kit (Roche, Mannheim, Germany) according to the manufacturer's instructions. In brief, $5 \times 10^{3}$ cells were seeded in 96-well plates and cultured overnight. Cells were treated with celecoxib $(0,1,2,4,8,16$ or $32 \mu \mathrm{M})$, PD184161 $(0,5$, $10,20,40,80$ or $160 \mu \mathrm{M})$ or a combination of the two drugs (both from Pfizer, Inc., New York, NY, USA). After 72 h, the cells were incubated with WST-1 reagent for $2 \mathrm{~h}$ at $37^{\circ} \mathrm{C}$. The absorbance (optical density; OD) was measured at $450 \mathrm{~nm}$ with an automated microplate reader (Model 550; Bio-Rad Laboratories, Inc., Hercules, CA, USA). The percent viability of cells in each group was calculated using the following equation: Cell viability $=$ mean OD of experimental group $/$ mean OD of control group x $100 \%$.

Isobologram analysis. Isobologram analysis provides a graphical presentation for the evaluation of combined drug effects, as described by Steel and Peckham (16). Briefly, the half maximal inhibitory concentration $\left(\mathrm{IC}_{50}\right)$ of celecoxib and $\mathrm{IC}_{50}$ of PD184161 were presented as 'a' $\left(0, \mathrm{IC}_{50}\right.$ of celecoxib) and ' $b$ ' $\left(\mathrm{IC}_{50}\right.$ of PD184161, 0) in a two-coordinate plot, and the line of additivity was constructed by connecting the two points. The concentrations of the two drugs used in combination produced new $\mathrm{IC}_{50} \mathrm{~s}$, which were denoted as 'c' and 'd' in the same plot. Points ' $c$ ' and 'd' that are below, at or above the isobologram line for a given effect level indicate synergistic, additive or antagonistic effects, respectively.

Mouse xenograft model. A total of $48 \mathrm{BALB} / \mathrm{c}$ female nude mice (weighing 20-25 g) were obtained from Shanghai SLAC Laboratory Animal Co., Ltd. (Shanghai, China) and acclimatized for 4 days. The mice were maintained in a temperature- and humidity-controllled room $\left(21^{\circ} \mathrm{C}\right.$ and $50 \%$ humidity) under a 12-h light/dark cycle with ad libitum access to standard food and water. GBC-SD and NOZ cells $\left(1 \times 10^{7}\right)$ were each subcutaneously injected into the flanks of the mice. At 2 weeks after tumor cell inoculation, the 


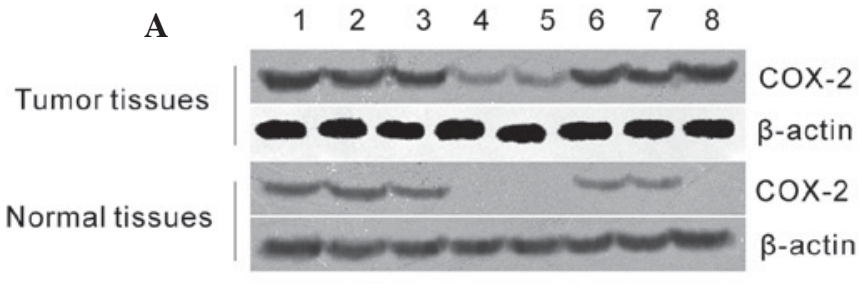

C

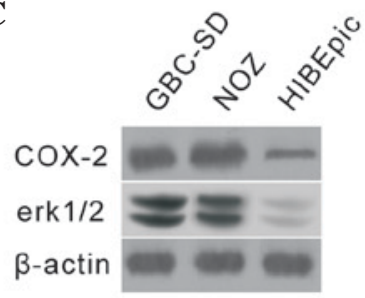

D

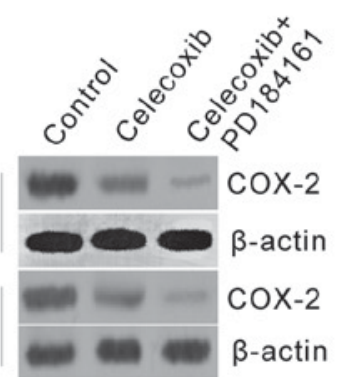

B

Tumor tissues

Normal tissues $\begin{array}{llllllll}1 & 2 & 3 & 4 & 5 & 6 & 7 & 8\end{array}$

Figure 1. Expression of COX-2 and p-ERK1/2 protein in GBC cell lines and tissues. (A) COX-2 and (B) p-ERK1/2 were upregulated in GBC tissues as compared with normal tissues. (C) COX-2 and p-ERK1/2 protein expression levels in GBC-SD and NOZ human GBC cells were higher than that in HIBEpiC human biliary epithelial cells. (D) Expression of COX-2 was decreased following treatment with celecoxib compared with the control (no treatment), and was decreased markedly more following treatment with a combination of celecoxib and PD184161. (E) Similar alterations were observed in p-ERK1/2 levels following treatment with PD184161 alone or in combination with celecoxib. COX-2, cyclooxygenase-2; p-ERK1/2, phospho-extracellular signal-regulated kinase 1/2; GBC, gallbladder carcinoma.

mice were treated with celecoxib (50 mg/kg/day), PD184161 (300 $\mathrm{mg} / \mathrm{kg} /$ day) or combination of the two drugs by oral administration for 24 days. Tumor size was measured every week. After 28 days, the mice were sacrificed by cervical dislocation and the xenografted tumors were weighed. Tumor volumes were determined according to the formula $\mathrm{L} \mathrm{x} \mathrm{W}^{2} / 2$, where $\mathrm{L}$ is the largest diameter of the tumor and $\mathrm{W}$ is the smallest diameter perpendicular to $\mathrm{L}$.

Apoptosis assay. Apoptosis in the cell lines was examined by flow cytometry using the Annexin V/fluorescein isothiocyanate (FITC) Apoptosis Detection kit (BD Biosciences, Franklin Lakes, NJ, USA). Cells were pretreated with $8 \mu \mathrm{M}$ celecoxib and $40 \mu \mathrm{M}$ PD184161 (alone or in combination) and then cultured in 6 -well plates. After $48 \mathrm{~h}, \geq 1 \times 10^{5}$ cells (including floating cells) were collected, centrifuged at $500 \times \mathrm{g}$ for $10 \mathrm{~min}$ at $4^{\circ} \mathrm{C}$, washed twice with cold PBS and resuspended in binding buffer. Double staining was performed with Annexin V/PI in a dark room at room temperature for $15 \mathrm{~min}$, and all samples were analyzed by flow cytometry within $1 \mathrm{~h}$. FlowJo software version 7.6.1 (Tree Star Inc., Ashland, OR, USA) was used to analyze the flow cytometry data.

Analysis of mitochondrial membrane potential $(\Delta \Psi m)$. $\Delta \Psi \mathrm{m}$ is an important parameter of mitochondrial function that may be used as an indicator of cell health (17). JC-1 (5,5',6,6'-tetrachloro-1,1',3,3'-tetraethylbenzimidazolocarbocyanine iodide) is a lipophilic, cationic dye that can selectively enter into mitochondria and reversibly change color from red to green as the membrane potential decreases. In healthy cells with high $\Delta \Psi \mathrm{m}, \mathrm{JC}-1$ spontaneously forms complexes known as J-aggregates with intense red fluorescence. By contrast, in apoptotic or unhealthy cells with low $\Delta \Psi \mathrm{m}, \mathrm{JC}-1$ remains in its monomeric form, which produces only green fluorescence.
Briefly, cells were pretreated with $8 \mu \mathrm{M}$ celecoxib and $40 \mu \mathrm{M}$ PD184161 (alone or in combination) for 2, 4, 6 or $8 \mathrm{~h}$, then adjusted to a density of $5 \times 10^{5} / \mathrm{ml}$, trypsinized and washed in PBS. The cells were resuspended in $1 \mathrm{ml}$ of complete medium, and stained with $5 \mu \mathrm{g} / \mathrm{ml} \mathrm{JC}-1$ (Beyotime Institute of Biotechnology, Shanghai, China) for $20 \mathrm{~min}$ at $37^{\circ} \mathrm{C}$ in total darkness. The cells were then washed twice and resuspended in PBS, and analyzed by flow cytometry.

Cell cycle analysis. Following treatment with $8 \mu \mathrm{M}$ celecoxib and $40 \mu \mathrm{M}$ PD184161 (alone or in combination) for $48 \mathrm{~h}$, cells were washed twice with ice-cold PBS, fixed in $70 \%$ cold ethanol, treated with $100 \mu \mathrm{g} / \mathrm{ml}$ ribonuclease A (Roche) and labeled with $50 \mu \mathrm{g} / \mathrm{ml}$ PI for $1 \mathrm{~h}$ at $37^{\circ} \mathrm{C}$. DNA content was subsequently analyzed by flow cytometry.

Statistical analysis. Data are presented as the mean \pm standard deviation of at least three independent experiments. Differences among variables were assessed by the Student's $t$-test for two groups and ANOVA for multiple groups. $\mathrm{P}<0.05$ was considered to indicate a statistically significant difference.

\section{Results}

Expression of COX-2 and p-ERK1/2 protein in GBC tissues and cell lines. Western blot analysis revealed that COX-2 and p-ERK1/2 protein were abundant in all GBC cases, while their expression levels were markedly lower or undetectable in normal tissues (Fig. 1A and B), indicating that COX-2 and p-ERK1/2 overexpression were frequent in GBC tissues. In addition, COX-2 and p-ERK1/2 protein levels were significantly higher in GBC-SD and NOZ cells than that in HIBEpiC cells (Fig. 1C and D), indicating that COX-2 and p-ERK1/2 may participate in the development of GBC. 

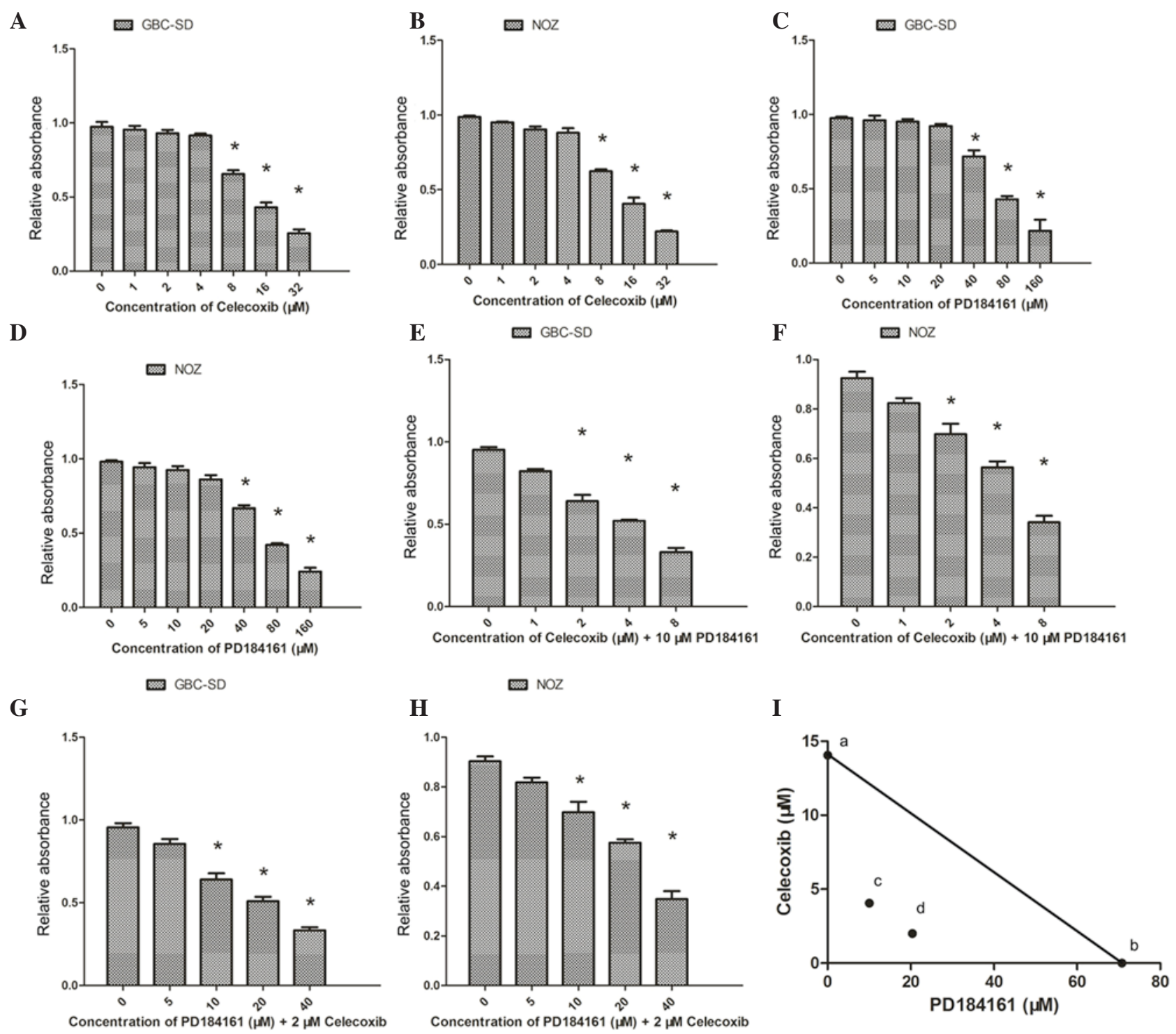

Figure 2. Celecoxib and PD184161 inhibited GBC-SD and NOZ cell growth. Celecoxib caused the concentration-dependent inhibition of (A) GBC-SD and (B) NOZ cell growth. Similarly, PD184161 caused the concentration-dependent inhibition of (C) GBC-SD and (D) NOZ cell growth. Combined treatment with $10 \mu \mathrm{M}$ PD184161 and 2-4 $\mu \mathrm{M}$ celecoxib significantly inhibited (E) GBC-SD and (F) NOZ cell proliferation compared with $10 \mu \mathrm{M}$ PD184161 single treatment; and combined treatment with $2 \mu \mathrm{M}$ celecoxib and 10-20 $\mu \mathrm{M}$ PD184161 significantly inhibited (G) GBC-SD and (H) NOZ cell proliferation compared with $2 \mu \mathrm{M}$ celecoxib single treatment. (I) Isobologram analysis indicated that celecoxib and PD184161 exert synergistic inhibitory effects on GBC cell growth, as data points 'c' and 'd' (indicating half-maximal inhibitory concentrations of the drugs used in combination) were positioned below the line that indicates an additive effect. ${ }^{*} \mathrm{P}<0.05$ vs. blank control group.

COX-2 protein levels were decreased in GBC-SD and $\mathrm{NOZ}$ cells following treatment with celecoxib, and p-ERK1/2 protein level was also markedly decreased following treatment with PD184161. Furthermore, compared to single treatments, COX-2 and p-ERK1/2 protein levels were markedly lower following treatment with a combination of celecoxib and PD184161 (Fig. 1E).

PD184161 and celecoxib co-inhibit the growth of GB-SD and NOZ cells. A WST-1 assay revealed that celecoxib inhibited the growth of GBC-SD and NOZ cells in a concentration-dependent manner; celecoxib at concentrations of $0-4 \mu \mathrm{M}$ did not significantly suppress the proliferation of GBC-SD and $\mathrm{NOZ}$ cells, but proliferation was significantly suppressed by concentrations of $8-32 \mu \mathrm{M}$ (Fig. $2 \mathrm{~A}$ and $\mathrm{B} ; \mathrm{P}<0.05$ ). For GBC-SD and NOZ cells, the calculated $\mathrm{IC}_{50}$ s were 14.05 and $12.42 \mu \mathrm{M}$, respectively. Similarly, PD184161 treatment led to a concentration-dependent inhibition of growth over the concentration range of 40-160 $\mu \mathrm{M}$ (Fig. 2C and D), with $\mathrm{IC}_{50} \mathrm{~s}$ of 70.79 and $66.77 \mu \mathrm{M}$ for GBC-SD and NOZ cells, respectively.

The inhibitory effects of combined treatment with celecoxib and PD184161 were also evaluated. As shown in Fig. 2E and $\mathrm{F}$, combined treatment with $2-4 \mu \mathrm{M}$ celecoxib and $10 \mu \mathrm{M}$ PD184161 (sub-effective doses) resulted in significant inhibition of cell growth in GBC-SD and NOZ cells as compared to cells treated with the same dose of PD184161 alone. Similarly, combined treatment with 10-20 $\mu \mathrm{M}$ PD184161 and $2 \mu \mathrm{M}$ celecoxib (sub-effective doses) resulted in significant inhibition of cell growth in GBC-SD and NOZ cells as compared to cells treated with the same dose of celecoxib alone (Fig. $2 \mathrm{G}$ and H).

Isobologram analysis revealed that the growth inhibitory effect of combined treatment with celecoxib and PD184161 
A

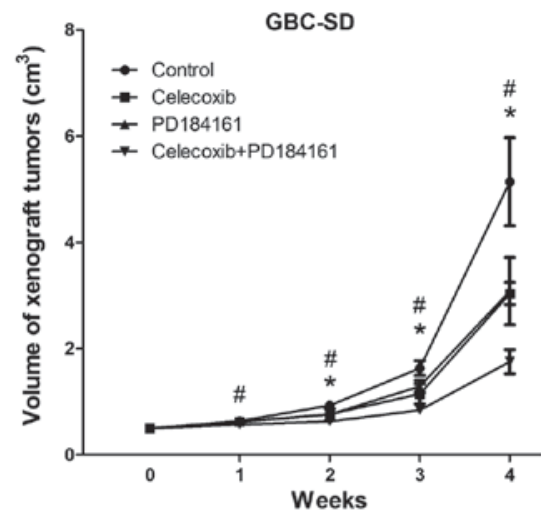

C

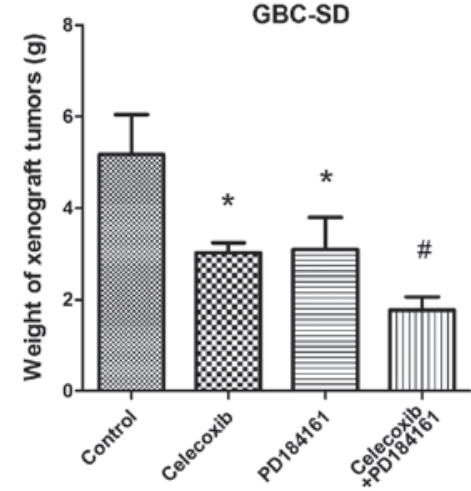

B

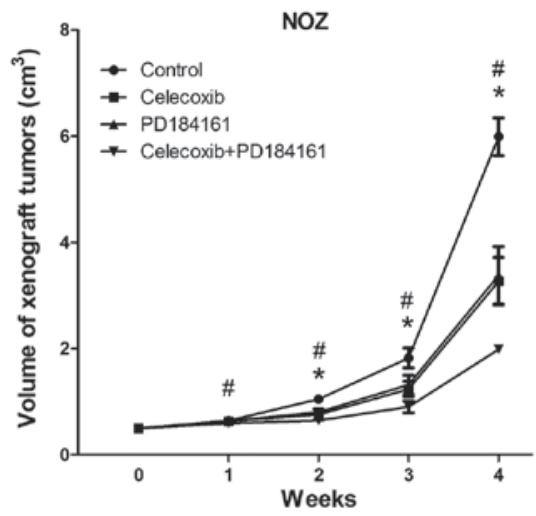

D

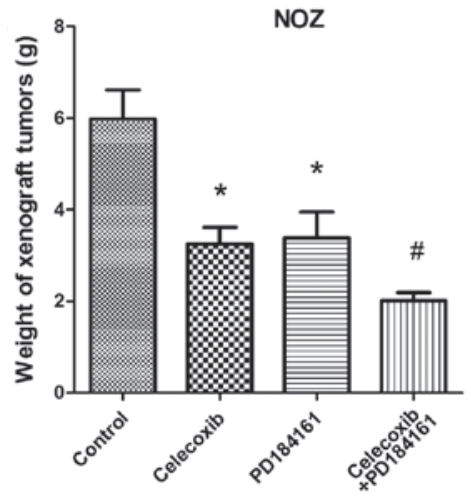

Figure 3. Celecoxib combined with PD184161 inhibited xenograft tumor growth. In (A) GBC-SD and (B) NOZ xenografts, tumor volumes in the co-treatment groups were significantly less than that of the single treatment and control groups. In (C) GBC-SD and (D) NOZ xenografts, tumor weights in the co-treatment groups were significantly less than that of the single treatment and control groups. Data are presented as the mean \pm standard deviation. "P<0.05 vs. the blank control group; ${ }^{\#} \mathrm{P}<0.05$, combined treatment group vs. all other groups.

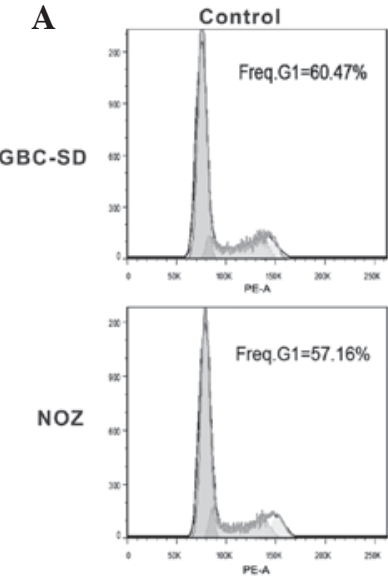

B

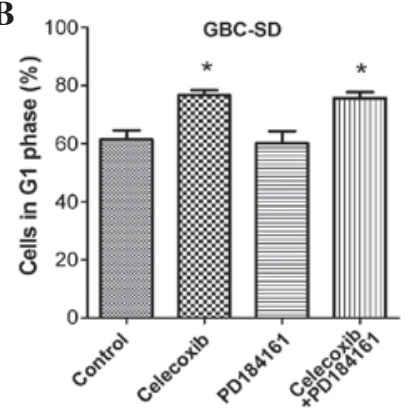

Celecoxib
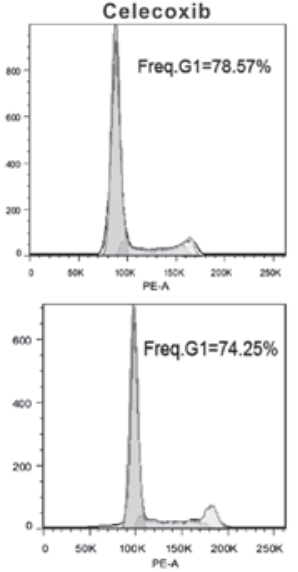

C

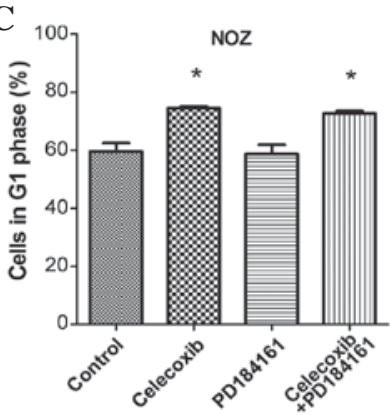

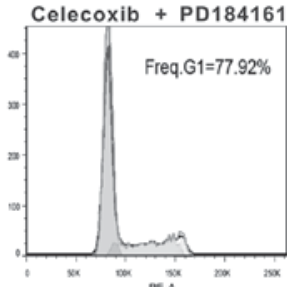

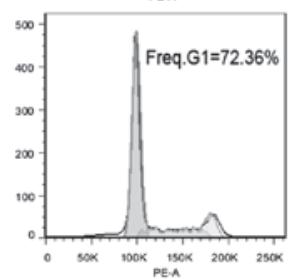

D

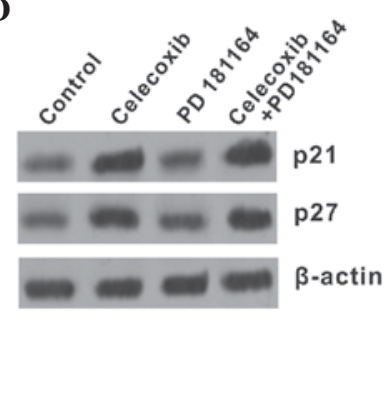

Figure 4. Celecoxib induced G1 phase arrest GBC-SD and NOZ cells. (A) Flowjo software was used for the analysis of flow cytometry data. The images shown are representative of three independent experiments. (B and C) The proportion of the G1 population increased significantly following treatment with celecoxib or combination treatment, whereas PD184161 had no significant effect on cell cycle distribution. (D) The expression levels of p21 and p27 in GBC-SD cells were significantly increased following treatment with celecoxib or combination treatment, whereas they did not change significantly following treatment with PD184161. Data are presented as the mean \pm standard deviation. ${ }^{*} \mathrm{P}<0.05$ vs. control. 
$\mathbf{A}$
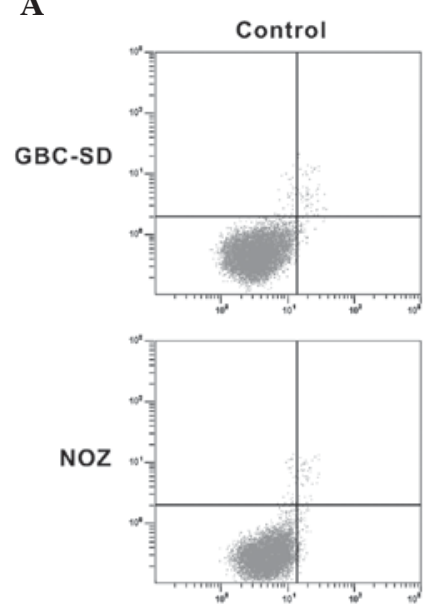

B
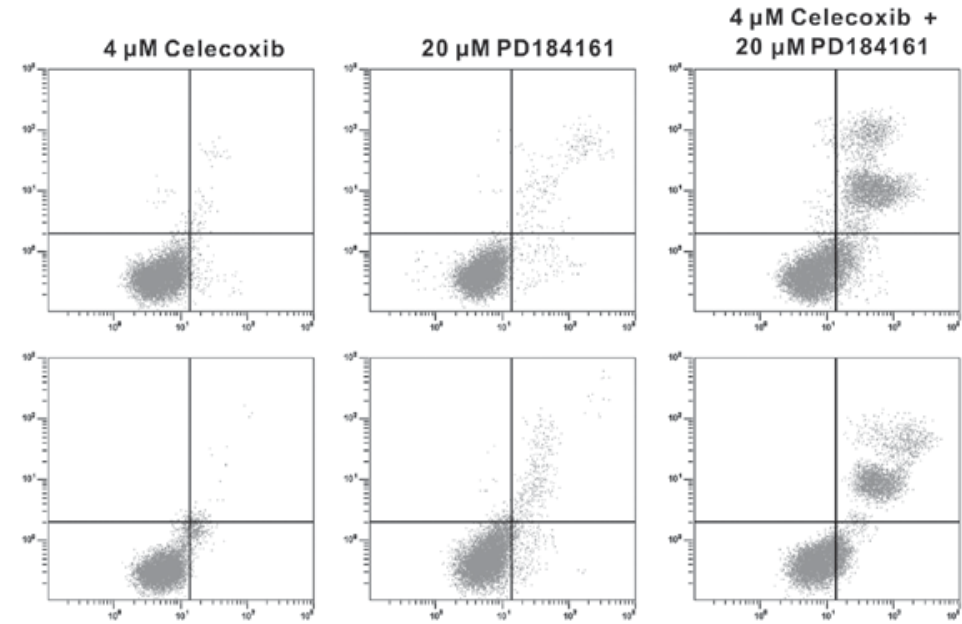

C

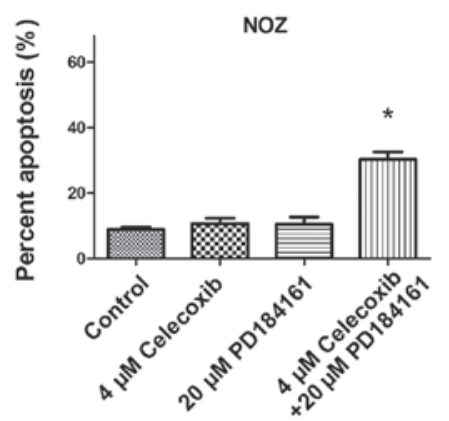

Figure 5. Combination of celecoxib and PD184161 induced apoptosis in gallbladder carcinoma cells. (A) Representative flow cytometry results from three independent experiments are shown. Lower left quadrants represent the Annexin ${ }^{-} / \mathrm{PI}^{-}$viable cells, lower right quadrants represent the Annexin ${ }^{+} / \mathrm{PI}^{-}$early apoptotic cells, and the right upper quadrants represent Annexin ${ }^{+} / \mathrm{PI}^{+}$late apoptotic cells. In (B) GBC-SD and (C) NOZ cells, the apoptotic cell population did not increase following treatment with $4 \mu \mathrm{M}$ celecoxib or $20 \mu \mathrm{M}$ PD184161 alone; however, it was elevated following treatment with a combination of $4 \mu \mathrm{M}$ celecoxib and $20 \mu \mathrm{M}$ PD184161. Data are presented as the mean \pm standard deviation. * $\mathrm{P}<0.05$ vs. other groups. PI, propidium iodide.

was synergistic, since the data points 'c' and 'd' were located well below the line defining an additive effect (Fig. 2I).

Celecoxib combined with PD184161 inhibits GBC-SD and NOZ cell growth in vivo. The application of celecoxib and PD184161 (alone or combined) was found to inhibit the growth of xenograft tumors. As shown in Fig. 3A and B, in the first week, the volumes of the xenograft tumors treated with celecoxib combined with PD184161 were significantly reduced compared with the other groups (control and celecoxib and PD184161 single treatments). From the second week, the volume of xenograft tumors treated with celecoxib or PD184161 alone were also significantly reduced compared with the control group $(\mathrm{P}<0.05)$. Similarly, the weights of xenograft tumors treated with celecoxib combined with PD184161 for 24 days were markedly less than that of the other groups $(\mathrm{P}<0.05$; Fig. 3C and D).

Celecoxib causes $G 1$ arrest by promoting p 21 and p27 expression. Flow cytometric analysis (Fig. 4A-C) revealed that celecoxib alone or the combination of celecoxib and PD184161 significantly increased the G1 population of GBC-SD and NOZ cells compared with the controls (both $\mathrm{P}<0.01$ ); by contrast, the proportion of cells in G1 phase did not alter following treatment with PD184161 alone. Western blotting (Fig. 4D) revealed that the expression levels of p21 and p27 were markedly elevated in GBC cells following treatment with celecoxib, whereas p21 and p27 expression did not change following treatment with PD184161 alone. These results indicate that the cell cycle was blocked by celecoxib but not by PD184161.

Combination of celecoxib and PD184161 induces cell apoptosis. Annexin V-FITC/PI staining revealed that $4 \mu \mathrm{M}$ celecoxib did not promote apparent apoptosis in GBC-SD or NOZ cells, nor did $20 \mu \mathrm{M}$ PD184161. However, the combination of $4 \mu \mathrm{M}$ celecoxib and $20 \mu \mathrm{M}$ PD184161 significantly induced apoptosis in the two cell lines (Fig. 5).

Celecoxib and PD184161 treatment induces the collapse of $\Delta \Psi m$ and activates caspase-3 protein. JC-1 staining (Fig. 6A-C) revealed that GBC-SD and NOZ cells maintained high $\Delta \Psi_{\mathrm{m}}$ in the absence of celecoxib or PD184161, whereas the majority of GBC-SD and NOZ cells exhibited a gradual decrease in $\Delta \Psi_{\mathrm{m}}$ following $2-8 \mathrm{~h}$ incubation with $4 \mu \mathrm{M}$ celecoxib and $20 \mu \mathrm{M}$ PD184161. Western blot analysis revealed that the amount of mitochondrial cytochrome $c$ was markedly decreased following treatment with celecoxib and PD184161, whereas the amount of cytosolic cytochrome $c$ was increased in GBC-SD and NOZ cells, indicating that celecoxib and PD184161 can promote the release of cytochrome $c$ from the mitochondria to the cytoplasm (Fig. 6D). Furthermore, levels of cleaved caspase-3 were markedly increased in GBC-SD cells following treatment with celecoxib and PD184161, indicating that caspase- 3 was extensively activated. 
A
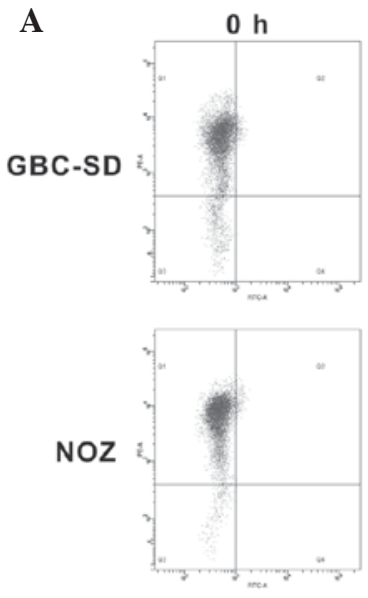

B

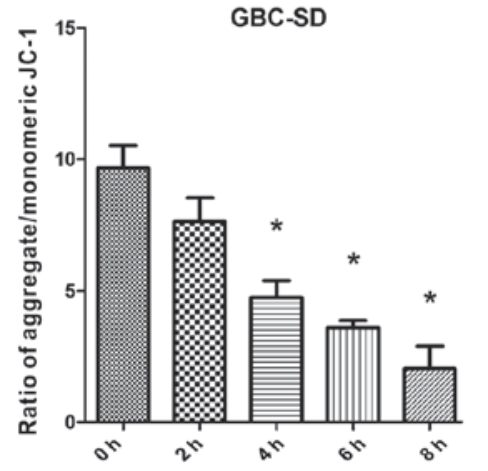

$2 \mathrm{~h}$
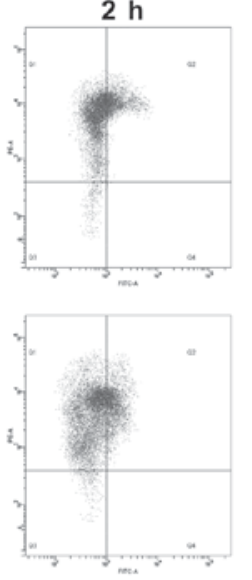

C
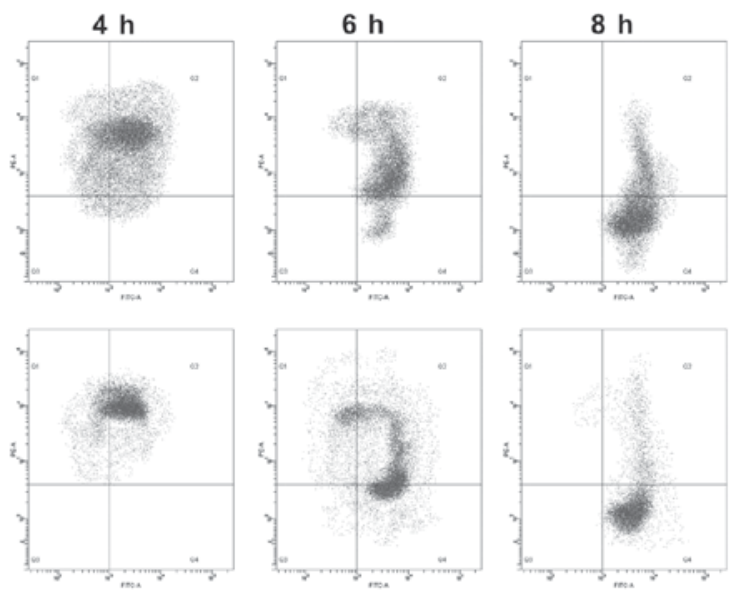

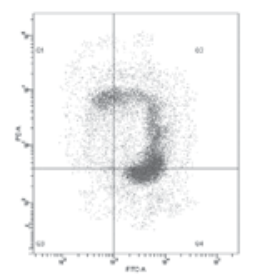

D

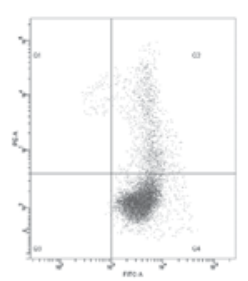

NOZ
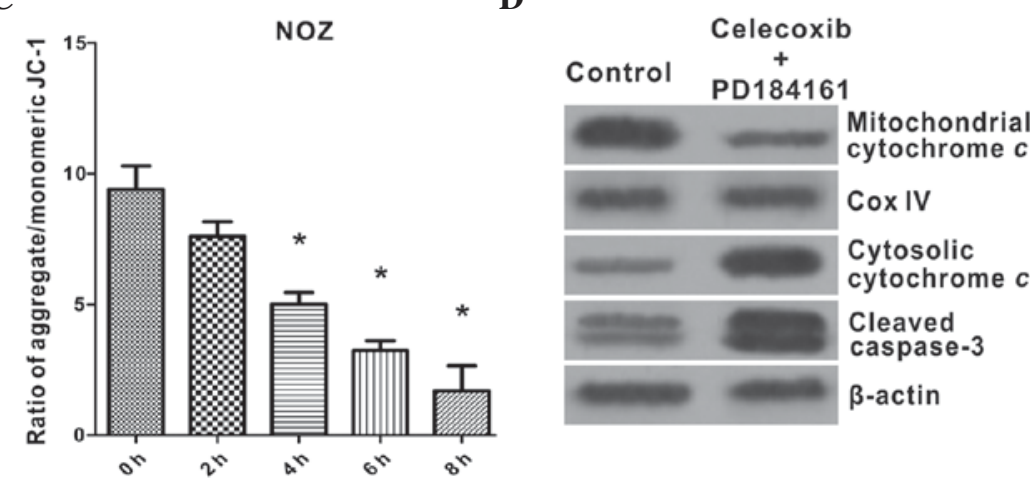

Figure 6. Celecoxib and PD184161 induced the collapse of the mitochondrial membrane potential. (A) Flow cytometric analysis was used to assess the ratio of red/green fluorescence (aggregate/monomeric JC-1) in GBC-SD and NOZ cells following treatment with celecoxib and PD184161. In (B) GBC-SD and (C) NOZ cells, following $2 \mathrm{~h}$ of treatment, the red fluorescence was significantly decreased while the green fluorescence was significantly increased, indicating a reduction in mitochondrial membrane potential. Data are presented as the relative ratio of the mean fluorescence intesnsity of aggregate (red) and monomeric (green) JC-1. (D) Western blot analysis indicated that treatment with celecoxib and PD184161 induced cytochrome $c$ release from mitochondria to cytoplasm and the activation of caspase- 3 . ${ }^{*} \mathrm{P}<0.05$ vs. $0 \mathrm{~h}$.

\section{Discussion}

To enhance the efficacy of treatment for GBC is an important issue that is yet to be resolved in clinical practice. Since tumors were established to be a gene-related disease, much attention has been paid to targeted drugs that are designed to directly or indirectly interfere with the vital molecules involved in tumor growth and development. An increasing number of targeted drugs have been developed and applied in clinical practice, and these are occasionally used as a first-line treatment for certain tumors (18). It has been demonstrated that COX-2 and pERK1/2 proteins, which are involved in tumor carcinogenesis and development, are aberrantly increased in various malignant tumors of the digestive tract, including hepatic carcinoma (19), gastric carcinoma (20), colon cancer (21) and pancreatic cancer (22). Therefore, they are considered to be important therapeutic targets for these tumor types, and the efficacy of the COX-2 inhibitor celecoxib and the ERK1/2 inhibitor PD184161 have been confirmed in recent studies $(23,24)$. However, the effects of combining celecoxib with PD184161 on GBC cell growth have not yet been reported. In the present study, COX-2 and p-ERK1/2 expression levels were examined in GBC cell lines for the first time, and their potential as therapeutic targets for GBC treatment was also evaluated. In addition, the effects of celecoxib and PD184161 on the growth, cell cycle and apoptosis of GBC cells were investigated.

COX-2 and p-ERK1/2 expression were found to be markedly lower in normal gallbladder tissues than in GBC tissues, and their expression levels were also lower in HIBEpiC cells than in GBC-SD and NOZ cells. These results were consistent with previous studies (9-11), indicating that aberrant COX-2 and ERK1/2 activation may serve key roles in GBC tumorigenesis, and verifying their potential as targets for GBC targeted therapy. As a member of the MAPK family of signal transduction molecules, ERK1/2 is involved in the process of cell growth, proliferation and differentiation. The ERK1/2 signaling pathway is often over-activated in various tumors, and p-ERK1/2 expression is abnormally high. COX-2 is an important rate-limiting enzyme in the synthesis of prostaglandin E2 (PGE2). COX-2/PGE2 can activate a variety of signaling pathways involved in tumor carcinogenesis and development, including ERK1/2 signaling pathways. Under physiological conditions, COX-2 protein expression is weak or absent in the majority of normal tissues (25); by contrast, under pathological situations, such as in tumors or inflammation, its expression may be induced by a variety of inflammatory cytokines, which are produced by tumors or their microenvironment. It has been documented that interleukin (IL)-1 (26), IL-8 (27), nuclear factor $\kappa \mathrm{B}(28)$ and hypoxia inducible 
factor $1 \alpha$ (29) can stimulate the expression of COX-2. Most of these factors are abundant in GBC tissues, which may be the reason for the aberrant activation of COX-2 and p-ERK1/2 in GBC tissues and cell lines.

Subsequently, the effects of celecoxib and PD184161 on the growth, cell cycle and apoptosis of GBC-SD and NOZ cells, which exhibit high COX-2 and p-ERK1/2 expression, were examined. A WST-1 assay revealed that celecoxib and PD184161 exert concentration-dependent inhibitory effects on the growth of GBC-SD and NOZ cells, and that their combination exerts synergistic inhibitory effects on cell growth. Furthermore, p-ERK1/2 and COX-2 protein expression were significantly downregulated following treatment with PD184161 and celecoxib alone, while co-treatment caused a more marked decrease in p-ERK1/2 and COX-2 protein expression compared with single treatment. PD184161 exerts its antitumor activities predominantly by inhibiting ERK1/2 activation, resulting in inhibition of proliferation, migration and invasion, as well as induction of apoptosis (30). By contrast, celecoxib exerts its antitumor activities predominantly by suppressing COX-2 protein expression and PGE2 synthesis (31). Recent studies demonstrated that celecoxib can block ERK1/2 phosphorylation, thereby blocking the cell proliferation signaling pathway mediated by ERK1/2 $(32,33)$; this indicated that, to a certain extent, celecoxib may enhance the PD184161-induced inhibition of ERK1/2 activity, and that this may be a reasonable mechanism by which celecoxib combined with PD184161 could cause synergistic growth inhibition. Additionally, the inhibitory effects of celecoxib combined with PD184161 in vivo were confirmed by xenograft tumor experiments in the present study, indicating their potential for clinical application in the future.

Flow cytometric analysis revealed that celecoxib induces G1 arrest, while PD184161 does not affect the cell cycle distribution. Furthermore, celecoxib induced the expression of the cyclin-dependent kinase inhibitors p21 and p27, while PD184161 did not alter their expression levels. In accordance with another study (34), the present results suggested that accumulation of p21 and p27 caused by celecoxib leads to G1 arrest.

The induction of apoptosis by celecoxib and PD184161 was also examined in the present study. FITC-Annexin V/PI staining revealed that the combination of celecoxib and PD184161 induced a significant increase in cell apoptosis. Additionally, a JC-1 staining assay revealed that celecoxib and PD184161 induced a collapse of the $\Delta \Psi \mathrm{m}$, and western blotting demonstrated that celecoxib and PD184161 promote cytochrome $c$ release from the mitochondria to the cytoplasm as well as caspase-3 activation.

In conclusion, COX-2 and p-ERK1/2 protein, which are aberrantly expressed in GBC tissues and cells, may serve as effective therapeutic targets for GBC treatment. The combination of celecoxib and PD184161 treatments leads to synergistic inhibition of GBC cell growth through triggering cell cycle arrest and apoptosis. It is hoped that this work will extend the investigation of celecoxib and PD184161 to a broader extent by offering new insight into the clinical application of the multi-targeted treatment of GBC. In future studies, clinical trials based on celecoxib and PD184161 alone or in combination for the treatment of GBC patients should be performed to further validate the effectiveness and safety of these regimens.

\section{Acknowledgements}

This study was supported by the Key Program of Foundation for Young Talents in Colleges and Universities of Anhui Province (Hefei, China; grant no. 2013SQRL053ZD).

\section{References}

1. Stinton LM and Shaffer EA: Epidemiology of gallbladder disease: Cholelithiasis and cancer. Gut Liver 6: 172-187, 2012.

2. Eslick GD: Epidemiology of gallbladder cancer. Gastroenterol Clin North Am 39: 307-330, ix, 2010.

3. Hundal R and Shaffer EA: Gallbladder cancer: Epidemiology and outcome. Clin Epidemiol 6: 99-109, 2014.

4. Levy AD, Murakata LA and Rohrmann CA Jr: Gallbladder carcinoma: Radiologic-pathologic correlation. Radiographics 21: 295-314, 2001.

5. Bonet Beltrán M, Allal AS, Gich I, Solé JM and Carrió I: Is adjuvant radiotherapy needed after curative resection of extrahepatic biliary tract cancers? A systematic review with a meta-analysis of observational studies. Cancer Treat Rev 38: 111-119, 2012.

6. Zhu AX and Hezel AF: Development of molecularly targeted therapies in biliary tract cancers: Reassessing the challenges and opportunities. Hepatology 53: 695-704, 2011.

7. Bottegoni G, Favia AD, Recanatini M and Cavalli A: The role of fragment-based and computational methods in polypharmacology. Drug Discov Today 17: 23-34, 2012.

8. Greenhough A, Smartt HJ, Moore AE, Roberts HR, Williams AC, Paraskeva C and Kaidi A: The COX-2/PGE2 pathway: Key roles in the hallmarks of cancer and adaptation to the tumour microenvironment. Carcinogenesis 30: 377-386, 2009.

9. Doval DC, Azam S, Sinha R, Batra U and Mehta A: Expression of epidermal growth factor receptor, p53, Bcl2, vascular endothelial growth factor, cyclooxygenase-2, cyclin D1, human epidermal receptor-2 and Ki-67: Association with clinicopathological profiles and outcomes in gallbladder carcinoma. J Carcinog 13: $10,2014$.

10. Li Q and Yang Z: Expression of phospho-ERK1/2 and PI3-K in benign and malignant gallbladder lesions and its clinical and pathological correlations. J Exp Clin Cancer Res 28: 65, 2009.

11. Jinawath A, Akiyama Y, Yuasa Y and Pairojkul C: Expression of phosphorylated ERK1/2 and homeodomain protein CDX2 in cholangiocarcinoma. J Cancer Res Clin Oncol 132: 805-810, 2006.

12. Vicent S, López-Picazo JM, Toledo G, Lozano MD, Torre W, Garcia-Corchón C, Quero C, Soria JC, Martín-Algarra S, Manzano RG and Montuenga LM: ERK1/2 is activated in non-small-cell lung cancer and associated with advanced tumours. Br J Cancer 90: 1047-1052, 2004.

13. Yeh JJ, Routh ED, Rubinas T, Peacock J, Martin TD, Shen XJ, Sandler RS, Kim HJ, Keku TO and Der CJ: KRAS/BRAF mutation status and ERK1/2 activation as biomarkers for MEK1/2 inhibitor therapy in colorectal cancer. Mol Cancer Ther 8: 834-843, 2009.

14. Wong DJL, Robert L, Atefi MS, Lassen A, Avarappatt G, Cerniglia M, Avramis E, Tsoi J, Foulad D, Graeber TG, et al: Antitumor activity of the ERK inhibitor SCH772984 [corrected] against BRAF mutant, NRAS mutant and wild-type melanoma. Mol Cancer 13: 194, 2014.

15. Reckamp KL, Koczywas M, Cristea MC, Dowell JE, Wang HJ, Gardner BK, Milne GL, Figlin RA, Fishbein MC, Elashoff RM and Dubinett SM: Randomized phase 2 trial of erlotinib in combination with high-dose celecoxib or placebo in patients with advanced non-small cell lung cancer. Cancer 121: 3298-3306, 2015.

16. Steel GG and Peckham MJ: Exploitable mechanisms in combined radiotherapy-chemotherapy: The concept of additivity. Int J Radiat Oncol Biol Phys 5: 85-91, 1979.

17. Smaili SS, Hsu YT, Carvalho AC, Rosenstoc TR, Sharpe JC and Youle RJ: Mitochondria, calcium and pro-apoptotic proteins as mediators in cell death signaling. Braz J Med Biol Res 36: 183-190, 2003. 
18. Wijesinghe $\mathrm{P}$ and Bollig-Fischer A: Lung Cancer Genomics in the Era of Accelerated Targeted Drug Development. Adv Exp Med Biol 890: 1-23, 2016.

19. Bae SH, Jung ES, Park YM, Kim BS, Kim BK, Kim DG and Ryu WS: Expression of cyclooxygenase-2 (COX-2) in hepatocellular carcinoma and growth inhibition of hepatoma cell lines by a COX-2 inhibitor, NS-398. Clin Cancer Res 7: 1410-1418, 2001.

20. He XP, Shao Y, Li XL, Xu W, Chen GS, Sun HH, Xu HC, Xu X, Tang D, Zheng XF, et al: Downregulation of miR-101 in gastric cancer correlates with cyclooxygenase-2 overexpression and tumor growth. FEBS J 279: 4201-4212, 2012.

21. Wendum D, Masliah J, Trugnan G and Fléjou JF: Cyclooxygenase-2 and its role in colorectal cancer development. Virchows Arch 445: 327-333, 2004.

22. Pomianowska E, Schjølberg AR, Clausen OP and Gladhaug IP: COX-2 overexpression in resected pancreatic head adenocarcinomas correlates with favourable prognosis. BMC Cancer 14 458, 2014.

23. Lev-Ari S, Strier L, Kazanov D, Madar-Shapiro L, Dvory-Sobol H, Pinchuk I, Marian B, Lichtenberg D and Arber N: Celecoxib and curcumin synergistically inhibit the growth of colorectal cancer cells. Clin Cancer Res 11: 6738-6744, 2005

24. Klein PJ, Schmidt CM, Wiesenauer CA, Choi JN, Gage EA, Yip-Schneider MT, Wiebke EA, Wang Y, Omer C and Sebolt-Leopold JS. The effects of a novel MEK inhibitor PD184161 on MEK-ERK signaling and growth in human liver cancer. Neoplasia 8: 1-8, 2006.

25. Chen WS, Wei SJ, Liu JM, Hsiao M, Kou-Lin J and Yang WK: Tumor invasiveness and liver metastasis of colon cancer cells correlated with cyclooxygenase-2 (COX-2) expression and inhibited by a COX-2-selective inhibitor, etodolac. Int J Cancer 91: 894-899, 2001.
26. Huang ZF, Massey JB and Via DP: Differential regulation of cyclooxygenase-2 (COX-2) mRNA stability by interleukin-1 beta (IL-1 beta) and tumor necrosis factor-alpha (TNF-alpha) in human in vitro differentiated macrophages. Biochem Pharmacol 59: 187-194, 2000.

27. Manna SK and Ramesh GT: Interleukin-8 induces nuclear transcription factor-kappaB through a TRAF6-dependent pathway. J Biol Chem 280: 7010-7021, 2005.

28. Choi YH, Jin GY, Li GZ and Yan GH: Cornuside suppresses lipopolysaccharide-induced inflammatory mediators by inhibiting nuclear factor-kappaB activation in RAW 264.7 macrophages. Biol Pharm Bull 34: 959-966, 2011.

29. He J, Wang M, Jiang Y, Chen Q, Xu S, Xu Q, Jiang BH and Liu LZ Chronic arsenic exposure and angiogenesis in human bronchial epithelial cells via the ROS/miR-199a-5p/HIF-1 $\alpha /$ COX-2 pathway. Environ Health Perspect 122: 255-261, 2014.

30. Kohno M and Pouyssegur J: Targeting the ERK signaling pathway in cancer therapy. Ann Med 38: 200-211, 2006.

31. Ferrandina G, Ranelletti FO, Legge F, Lauriola L, Salutari V, Gessi M, Testa AC, Werner U, Navarra P, Tringali G, et al: Celecoxib modulates the expression of cyclooxygenase-2, ki67, apoptosis-related marker, and microvessel density in human cervical cancer: A pilot study. Clin Cancer Res 9: 4324-4331, 2003.

32. Li F, Liu S, Ouyang Y, Fan C, Wang T, Zhang C, Zeng B, Chai Y and Wang X: Effect of celecoxib on proliferation, collagen expression, ERK1/2 and SMAD2/3 phosphorylation in NIH/3T3 fibroblasts. Eur J Pharmacol 678: 1-5, 2012.

33. Li F, Fan C, Zeng B, Zhang C, Chai Y, Liu S and Ouyang Y: Celecoxib suppresses fibroblast proliferation and collagen expression by inhibiting ERK1/2 and SMAD2/3 phosphorylation. Mol Med Rep 5: 827-831, 2012.

34. Liu H, Huang P, Xu X, Liu J and Guo C: Anticancer effect of celecoxib via COX-2 dependent and independent mechanisms in human gastric cancers cells. Dig Dis Sci 54: 1418-1424, 2009. 